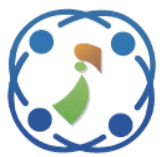

\title{
Quad Convolutional Layers (QCL) CNN Approach for Classification of Brain Stroke in Diffusion Weighted (DW) - Magnetic Resonance Images (MRI)
}

\author{
Andi Kurniawan Nugroho ${ }^{1,4}$ \\ Terawan Agus Putranto ${ }^{2}$ \\ Mauridhi Hery Purnomo $1,3,5 *$ \\ I Ketut Eddy Purnama1,3 \\ ${ }^{l}$ Electrical Engineering Department, Institut Teknologi Sepuluh Nopember, Surabaya, Indonesia. \\ ${ }^{2}$ RSPAD Gatot Subroto Presidential Hospital, Jakarta, Indonesia. \\ ${ }^{3}$ Department of Computer Engineering, Institut Teknologi Sepuluh Nopember, Surabaya, Indonesia. \\ ${ }^{4}$ Electrical Engineering Department, Universitas Semarang, Semarang, Indonesia. \\ ${ }^{5}$ University Center of Excellence on Artificial Intelligence for Healthcare and Society (UCE AIHeS), Indonesia. \\ * Corresponding author's Email: ketut@ee.its.ac.id; hery@ee.its.ac.id
}

\begin{abstract}
Commonly, clinicans have problems for recognising brain stroke injury images. However, with the advantages of Information technology it is expected that will be a new method that can support the clinicans' opinion for recognising the brain stroke injury for type of stroke (hemorrhagic, ischemic, and normal). Therefore, this study aim is to discovery a new model to classify hemorrhagic, ischemic and normal based on Diffusion Weighted (DW)Magnetic Resonance (MR) images. This study argues by using Qual Convolutional Layers (QCL-CNN) which applied in CNN can classified type of stroke. For this study experiment, this research conducted two experiment to asses the performance of QCL-CNN. The first experiments partitioned the MR image dataset into 20 percent testing and 80 percent training sets. Then, the second testing performed ten-fold cross-validation on the image dataset. The result from the first experiment of the classification accuracies obtained 93.90 percent $\left(1^{\text {st }}\right.$ dataset) and 94.96 percent $\left(2^{\text {nd }}\right.$ dataset). As for the second experiment, the results shows that the classification accuracies obtained 95.91 percent $\left(1^{\text {st }}\right.$ data set) and 97.31 percent ( $2^{\text {nd }}$ data set). The data source for this study gained from Indonesian hospital and the web sources dataset public from Ischemic Stroke Lesion Segmentation (ISLES). This study also compared, the QCL-CNN model with other architecture model such as AlexNet, ResNet50, and VGG16. The result of the comparison experiment shows that QCL-CNN architectures model has excellent performance than the others model.
\end{abstract}

Keywords: QCL-CNN, DW-MRI, Brain stroke injury, Image, Preprocessing, Classification.

\section{Introduction}

For the brain stroke injury, health staff generally used radiological modality of Computerized Tomography Scanning (CT scan), for examining the patient. The health staff identified stroke based on the caused of the brain stroke injury. Based on causes, stroke is classified into two, namely ischemic (in which the blood supply stops flowing to the brain due to blockage) and hemorrhagic (where there is bleeding in the brain tissue) [1]. Based on the classification, the health staff is essential to carry out an appropriate diagnosis before starting stroke treatment due to different disease conditions. It is due to an appropriate health staff diagnosis for the brain stroke injury lead to the appropriate and proper treatment for the patient. The treatment and diagnosis of stroke are carried out by clinical examination, followed by assessing radiological modalities, such as CT scan [2].

CT is the primary mode of diagnosis in the early stages of stroke for separating hemorrhagic from ischemic disorders. Nevertheless, CT has less ability for detect the stroke lesions during the patient's acute period. Conventional CT or Medical Resonance Image (MRI) occasionally ineffective at predicting the presence and amount of acute damage [3].

To address for ineffective of CT and MRI, the health staff using Diffusion Imaging as hyper- 
intensity (DWI).The reduction in water diffusion is reflected in the DW-MRI mode by a drop in the Apparent Diffusion Coefficient (ADC) trace map [4], [5], which is visible in Diffusion Imaging as hyperintensity (DWI). The usage of DWI has been proven in previous animal research, and the investigations showed, the DWI has an ability of showing ischemic brain alterations within five minutes and one to three hours after the patients feel the symptoms brain stroke injury. In medical diagnosis for human, these alterations are noticed as early as two to six hours after the beginning of brain stroke injury symptoms. Moreover, DWI has a low rate of false-negative investigations (5\%), with a clear distinction between ischemic and hemorrhagic lesions. Thus, DWI enables the early identification of the kind, location, and size of a brain stroke and aids in the prediction of the patients' clinical outcomes [6].

To address the problem, the hospital create a procedures, for the clinicans treating the patient's brain stroke injury. Firstly, before spotting a stroke, clinicians need to confirm the symptoms with the patient or the patient's family members. This identification process is essential since it helps the clinician to have clearly and accurately clinical'sjudgement for determining the patient's condition.

Next, the clinicians is analyzing the neurological imaging recordings of the patient using the CT scan. By using the CT scan, it is the first technique for diagnosing brain stroke. The reason for using The CT scan for detecting the brain stroke injury is the tool is affordable in price compare to the others medical tools.

Then, the patient brain's image is sent to the radiologist. The radiologist will determine the patient brain stroke injury type.

To support the radiologist decision, another tools used which called MRI. MRI is used by the radiologist to obtain detailed changes in patient's brain structure anatomy. With the advantages of technology DW mode applied in MRI to help detect the beginning of a patient's brain stroke injury, particulary for the brain stroke injury with the ischemic type.

After, the first patient's physical examination is performed. The clinicians give the patients treatmentent according to the clinical procedure.

However, the patients with brain stroke injury, is not only once patient. The hospitals are receveid numerous patients with stroke symptoms on the same day. It also become problematic for the hospital clinician to provide a suit treatment for patient's brain stroke injury if to many patient that need to be handle by the clinican
Brain strokes injury is caused by hemorrhage occur when a blood artery rupture and spills into the surrounding brain tissue. Hypertension, trauma, aberrant blood arteries (for instance, arteriovenous malformation (AVM)), bleeding problems, aneurysms, and drug use all contribute to this. Ischemic stroke, on the other hand, happens when the brain's blood supply is cut off due to a clot. After an ischemic stroke, a brain hemorrhage can occur, resulting in significant consequences [7].

Fig. 1 illustrates a human brain stroke injury image. The first ( a) and second (b) row indicate hemorrhage and ischemic stroke, respectively, whereas the third (c) row normal instances. The part of the brain where a brain stroke injury develops is highlighted in red's colours and it is to assist inexperienced readers. The intensity of the core infarct is what differentiates suspected hemorrhagic and ischemic strokes. Additionally, bleeding is indicated with darker in color than the ischemic core.

Based on the problem, the prior research showed that Computer-Aided Diagnosis (CAD) system can help supporting the manual diagnostic procedure in hospital in identifiying patient with brain stroke injury. However, in fact the doctor and clinicans rarely used the CAD system for diagnosing the patient with brain stroke injury [8,9]. So, , the researcher Peixoto and Filho [8] suggested further studies to automate the CAD system's performance.

From the prior research, there are a litte gap for research in detection in brain stroke injury image using DWI- MRI. Since, there was no research that employs the DWI- MRI for data set for experiment in detection of brain stroke injury. Futhermore, there was no research that suggesting method to detect brain stroke injury using DW-MRI.

Therefore, this paper proposed a new method to detect brain stroke injury on DW-MRI. As many research that already used deep learning to aid the study of medical image processing. This study also seek the possibility for using deep learning to slasify the brain stroke injury on DW-MRI.

Deep learning is a technique that support in a classification techniques. [10,11]. Deep learning also support an automation for calculating deep convulational system [11]. The key advantage of this methodology is that it outperforms other picture classification techniques. [12]. Since the beginning of development, numerous deep learning methods have been formed, for intances recurrent neural networks, Long Short-Term Memory (LSTM) [13], CNN [14], and Deep Belief Net (DBN) [15], all of which are based on the neural network concept. In addition to deep learning methods, other classification algorithms [16, 17, 18]. Although SVM works 
properly on linear data, it is difficult to process highdimensional information $[19,20]$.

Meanwhile, $\mathrm{KNN}$ is a simple method that requires a large storage capacity to accommodate millions of objects in the dataset [20]. When training CNN models with larger datasets, deeper architectures are recommended over shallow architectures. Bansal et al. [21]. However, this study also found that for larger datasets that used to performs the experiment is , shallow architectures outperform deeper architectures.

In this study, a stroke classification method was proposed Quad Convolutional layers (QCL-CNN) for stroke classification on DW-MRI images, using the $\mathrm{CNN}$ architecture comprising two main blocks, each consisting of two convolutions with max-pooling. The goal is that reduction in size causes less computational overhead for subsequent network layers and prevents excessive over-fitting. Moreover,an automatic classification method was proposed in this study to predict the category of DWMRI brain images, including hemorrhage, ischemic, and normal categories.

The contributions of this study are :

- Classifying the particular features of brain stroke injury using DW-MRI imaging.

- Adapting CNN architecture with QCL to produce shallow architure to gain high accuracy brain stroke injury classification.

This paper further ordered as follows: The Section 2 discusses the preliminary studies that similar with the QCL-CNN, Section 3 explains the classifies the proposed methodology for this study, and Section 4 provides details of the experiment to test the QCLCNN with MR image datasets and the results. Meanwhile, the Section 5 discusses the result of the QCL-CNN and Section 6 summarises the result of this study.

\section{Preliminary studies}

This section explores the preliminary studies related to an approach of brain classification. First, the study conducted by Saatman et al. [22]. This study classified traumatic brain injury to seek the best treatment for patient. This paper used the degree of brain injury to classify the traumatic of brain injury. Saatman et.al[22] research employs the Glasgow Coma Scale (GCS) and other criteria. However, this study only used the expert judgement from health staff to clasify the brain injury. Therefore, this study has a weakness on the data accuration. The data is only used the health staff opinion.
Then, Mosqueda et al. [23] examined the concept of clinical data classification on acute ischemic patients using CT or MR angiography images to detect large and small strokes (Boston Acute Stroke Imaging Scale/ BASIS). This research argues, that BASIS classification instrument is effective and appears superior to Alberta Stroke Program Early CT Score (ASPECTS) in predicting outcomes in acute ischemic stroke. However, the ASPECTS has weakness because this method employs scoe based on the health staff opinion.

Next, S. Anbumozhi [24] developed a technique for detecting and diagnosing brain stroke. A directional filtering method is used to minimize impulse noise in brain MRI pictures. Oriented local histogram equalization (OLHE) approaches are used to improve the quality of the noise-reduced brain image. After that, the skull is eliminated from the improved brain image. A k-means classifier is used to extract features and segment the stroke region. Based on the location, a segmented stroke is classified as mild, moderate, or severe. Nevertheles, this OLHE only detects small infact in brain stroke injury.

Other researchers, Gautam et al. [25] illustrate how to segment hemorrhagic strokes from CT scan images using a fuzzy clustering variation called Modified Robust Fuzzy C-Means Clustering (MRFCM) and a segmentation technique called Distance Regularization Level Set Evolution (DRLSE). However, this approach was unable to identify a very small lesion from a CT scan image. Similar with the first experiment conducted by Gautam et al [26], also presented a system for classifying CT scan images of the brain into three categories: hemorrhage, ischemia, and normal. Local Gradient of Gradient Pattern was offered as a new feature descriptor (LG2P). Using 900 image datasets with fine $\mathrm{kNN}$ and cubic SVM, the greatest classification accuracy of 83.11 percent and 86.11 percent was attained. However, this precision was insufficient and needed to be improved in order to achieve better outcomes.

From the explanation on the prior studies above [22-26], it can be summarized that none of them doing research to classify ischemic stroke, stroke haemorrhage, and normal conditions of the human brain using deep learning methods with limited layer convolutions. Therefore, the research in this paper is the first to proposes the use of four-CNN convolution layers in deep learning for brain stroke classification, and the author's name it as Quad Convolutional Layers (QCL)-CNN. 


\section{Proposed method}

\subsection{Dataset for the experiment/ preparation}

The study used DW MRI scans from two hospitals in Indonesian and the web sources dataset public from Ischemic Stroke Lesion Segmentation (ISLES) to gather 1742 hemorrhage, acute ischemic, and normal strokes for every class. This expemerint employed the 5226 DW MR images.

This research worked based on the ethical approval from Health Research and Development Agency Indonesia (Badan Penelitian dan Pengembangan Kesehatan - Balitbangkes) No. LB.02.01/2/KE.289/2018. Moreover, all clinical images were labelling by number, so the patients' identity did not recognised in this study.

The MRI were acquired with the following parameters: field strength $1.5 \mathrm{~T}$, slice thickness $5 \mathrm{~mm}$, slice distance $0.7 \mathrm{~mm}$, pixel size $320 \times 320$, echo duration $71 \mathrm{~ms}$, repetition time $4000 \mathrm{~ms}$, flip angle $150^{\circ}$, and step coding phase 287. All DW-MRI images were labeled appropriately for acute ischemic, hemorrhagic, and normal strokes.

\subsection{Data preparation and data augmentation}

Each DWI scan is limited in terms of the number of lesions. Additionally, whether training data were created at the cut-out level or lesion sample stage, only a small number of patches were accessible. Due of the high number of parameter files and the requirement to generate a large number of pictures (patches), different methods were used to replenish the training data based on a finite amount of DWI.

To begin, the extracted picture (patch) was split, zoomed, and flipped horizontally. Second, the patch extraction procedure was also used to supplement data. 324 DWI were utilized for training, 36 for validation, and 90 for testing in the initial data set.

The solution for this study, the researcher employs the data augmentation.

The algorithm can seen below,

Algorithm: Pseudocode for preparing the data and augmentation

1. Load the original input image from the disk.

2. The original image is changed randomly with the technique of sliding series, series, zoom, flip horizontally.

3. images that have been processed, used and written back to disk.

4. the second and third steps are repeated continuously according to the number of $\mathrm{N}$
Numerous preprocessing processes were conducted for this experiment the images were attained using a variety of scanners and protocols. Therefore, this study applied homogeneous linear sampling method to gain DW-MRI Image in uniform physical size.

Moreover, because the image is anisotropic in the axial (or z-axis) direction, the resampling operation introduced interpolation errors. As a result, a 2D slice analysis was done rather than a 3D volume study.

Data preparation and data augmentation was done to eliminate superfluous contextual information and to equalize the pixel counts of normal and lesion pixels. As a result it is an effective form of data augmentation, as all pixels were classified as being part of the obstruction and bleeding. A patch was extracted around each of these pixels and placed in random spots. Each patch contains pixels from infarct/hemorrhage and general tissue/background. When a pixel was located in the center of an infarct/bleed, the patch extracted included only that pixels.

\subsection{Pre-processing}

A multi-layer design was proposed to perform the brain's DW-MRI classification. In this experiment, a 2D slice of the DW-MRI image was considered on the section's axial side. The hospital radiologist ( as expert fo brain stroke injury) is help aid this study in the process of labelling and selecting the images to be separated into three classes. The classes of images was grouping to hemorrhage, ischemic, and normal.

\subsection{Image classification process}

$1^{\text {st }}$ Phase, is input Layer, which consist of training, validating, and testing data.

$2^{\text {nd }}$ Phase is Convolution extracts features from images by convolving each element with a filter that has the same depth as the image[27]. The last detail before implementing CNN defined the end-to-end design and the Convolutional layer's dimensions to construct the building blocks mentioned above.

To calculate the spatial dimensions of the Convolutional Layer, a formula that functions from the input volume and hyperparameters is needed as follows:

For each $\left(W_{\text {out }}(i)\right)$ input volume dimension:

$$
W_{\text {out }}(i)=1+\frac{W_{\text {In }}(i)-R+2 P}{S}
$$

Where, $W_{I n}(i)$ is the input dimension, $\mathrm{R}$ is the value of the receptive field, $\mathrm{P}$ is the padding value, 

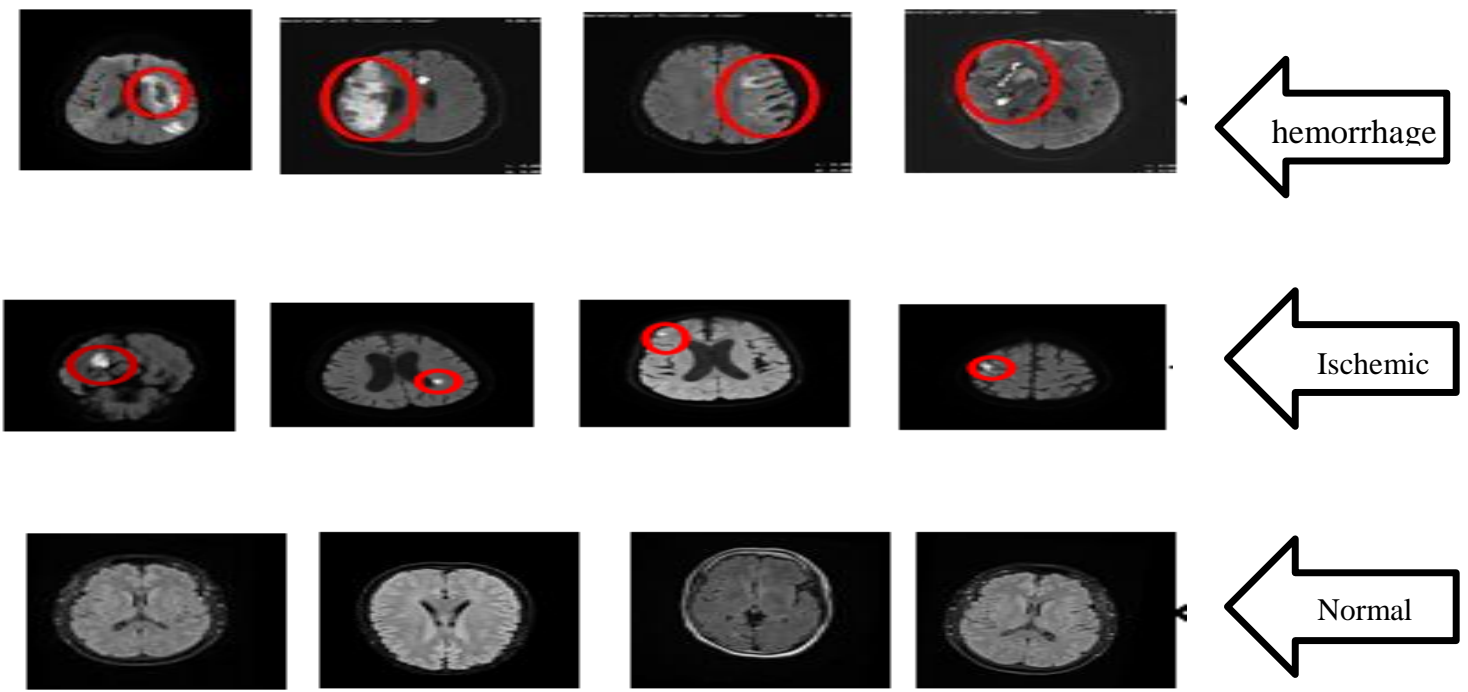

Figure. 1 DWI mode that differentiates hemorrhage, ischemic and normal conditions

and $\mathrm{S}$ is the stride value. This formula does not depend on the depth of the input. Therefore, to obtain the volume, the dimension value is explained in the following steps:

Suppose the input volume dimension is $224 \times 224 \times 3$, and the stride value is 2 along with the horizontal and vertical directions, then, WIn $=224$ and $\mathrm{S}=2,(2 . \mathrm{P}-\mathrm{R})$ need to be the integer for the calculated value. When the padding is 0 and $R=4$, the results obtained are Wout $=(224-4+2.0) / 2+1$ $=284 / 2+1=111$.

$3^{\text {rd }}$ phase The third layer is ReLU. ReLU layer is to the Unit Rectifier, the most commonly used activation function for $\mathrm{CNN}$ neuron output. It is mathematically, explained as follows:

$$
f(x)=\left\{\begin{array}{cc}
x & x \geq 0 \\
0 & x<0
\end{array}\right.
$$

Unfortunately, the ReLu function is indistinguishable by origin, making it challenging to use with backpropagation training. Therefore, it was replaced with a smoothed version called the Softplus function:

$$
f(x)=\ln \left(1+e^{x}\right)
$$

The derivative of the soft plus function is sigmoid.

$$
\begin{aligned}
f^{\prime}(x)=\frac{d\left(\ln \left(1+e^{x}\right)\right)}{d x} & =\frac{e^{x}}{1+e^{x}} \\
& =\frac{1}{1+e^{-x}}
\end{aligned}
$$

The $4^{\text {th }}$ phase Pooling Layer . Convolutional is placed before the Pooling Layer, serves to reduce the spatial dimensions (Width $x$ Height) of the Input Volume. this does not affect the volume depth dimension. Down sampling is done at layer 4 . Reducing the size will also cause a reduction in information.

The $5^{\text {th }}$ phase is Dropout layer. This is a regularization strategy for preventing network overfitting [28].

The $6^{\text {th }}$ phase layer is Fully-Connected. n layer 6 , it functions as an output link and a layer that regulates the amount of output. The number of inputs for the 6th layer is multiplied by the matrix, and the bias vector is added.The range [ $\left[\begin{array}{ll}0 & 1\end{array}\right]$ [27] is then normalized using layer softmax.

In Fig. 2, this study proposes the QCL-CNN architecture, where there are convolution, maxpooling, and classification layers. Extraction features used consist of $\operatorname{conh} 2 \times 2.32$; $\operatorname{conh} 2 \times 2,32$; conv $2 \times 2$, 64 ; conv $2 \times 2,128$; and the presence of a $2 \times 2$ maxpooling layer, as well as making use of the embedded RELU activator between them.. The feature map is the output of the convolution and max-pooling operations in $2 \mathrm{D}$. The size of the feature map can be seen as follows $223 \times 223 \times 32,110 \times 110 \times 32$, $54 \times 54 \times 64$, and $26 \times 26 \times 128$. As for the convolution operation, it is $111 \times 111 \times 32,55 \times 55 \times 32,27 \times 27 \times 64$, and $13 \times 13 \times 128$ are the feature map sizes of the pooling operation, respectively. The input image used is $224 \times 224 \times 3$ in size.

Adam's algorithm is used to create the QCLCNN Model. And then, the QCL-CNN model will be utilized for image classification. Next, the model is used to classify the images on the test set. 
Table 1. Properties of QCL-CNN

\begin{tabular}{|c|c|c|}
\hline Numbers & Layer & Properties \\
\hline 1 & Input Layer & 1st layer of input size $224 \times 224 \times 3$ \\
\hline 2 & Convolution Layer & $1^{\text {st }}$ layer contains 32 filters of $[2,2]$ \\
\hline & & $3^{\text {rd }}$ layer contains 32 filters of $[2,2]$ \\
\hline & & $5^{\text {th }}$ layer contains 64 filters of $[2,2]$ \\
\hline & & $7^{\text {th }}$ layer contains 128 filters of $[2,2]$ \\
\hline 3 & ReLU Layer & ReLU is used in $1^{\text {st }}, 3^{\text {rd }} 5^{\text {th }}, 7^{\text {th }}, 10^{\text {th }}$. \\
\hline 4 & Max Pooling Layer & $\begin{array}{l}2 \times 2 \text { max pooling with stride[2 } 2] \text { has been used } \\
2 \text { th }, 4 \text { th }, 6^{\text {th }}, 8^{\text {th }} .\end{array}$ \\
\hline 5 & $\begin{array}{l}\text { Dropout Layer } \\
\text { Fully Connected Layer }\end{array}$ & $\begin{array}{l}11^{\text {th }} \text { er with dropout probability } 0.5 \\
12^{\text {th }} \text { layer with } 512 \text { output value and } 3 \text { or } 2 \text { output } \\
\text { value-dependent of the dataset }\end{array}$ \\
\hline & Softmax layer & 12th layer \\
\hline 6 & Classification Layer & $12^{\text {th }}$ layer for image classification \\
\hline
\end{tabular}

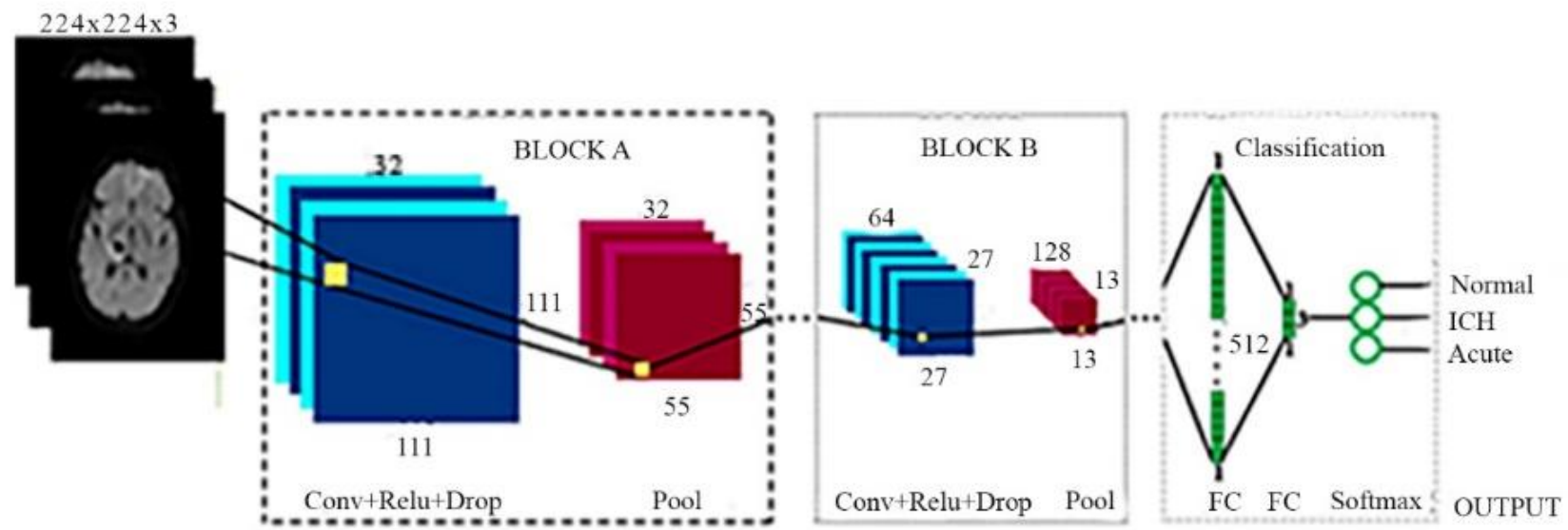

Figure. 2 Proposed research block diagram

The batch size used for the AlexNet proposal and network is 128, while for ResNet50, it is 12 .

\subsection{Implementation details and performance measures}

The experiment was carried out in 2 methods. In the first, the hemorrhage and ischemic data were divided into 80percent training and 20percent testing. The second experiment added normal DWMRI data by dividing it into 20percent testing and 80 percent training. The image preprocessing techniques were used to experiment by adding the data limitations with augmentation and image resizing methods at the beginning of the input image from the CNN process. Based on the trained model, the recommended QCL-CNN model were placed on the training dataset. As illustrated in Fig. 2 , it delivers classification results on the test dataset. The properties of all layers used are shown in Table 1. Furthermore, the following precisions were used to check the method's effectiveness: True Positive
Rate (TPR), false-positive rate (FPR), F-measure, accuracy, and ACC as an evaluation measure. This is calculated as follows:

$$
\begin{gathered}
\text { Precision }=\frac{T P}{T P+F P} \\
T P R=\frac{T P}{T P+F N} \\
F P R=\frac{F P}{F P+T N} \\
F_{\text {measure }}=2 \cdot \frac{\text { Precision } x T P R}{\text { Precision }+T P R} \\
\text { ACC }=\frac{T P+T N}{T P+T N+F P+F N}
\end{gathered}
$$

FN, FP, TN, and TP are False Negative, False Positive, True Negative, and True Positive. 


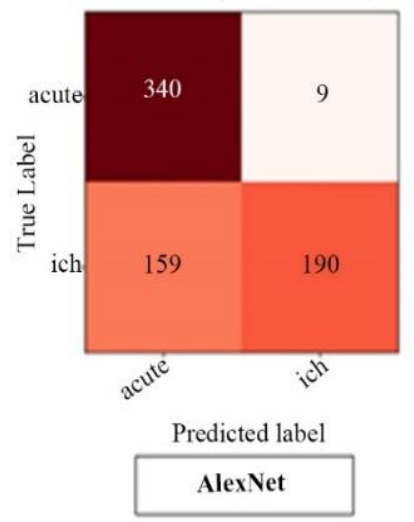

(a)

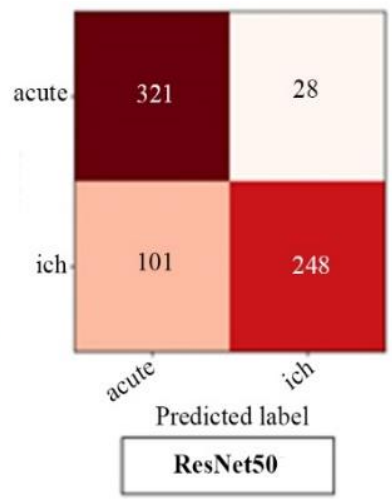

(b)

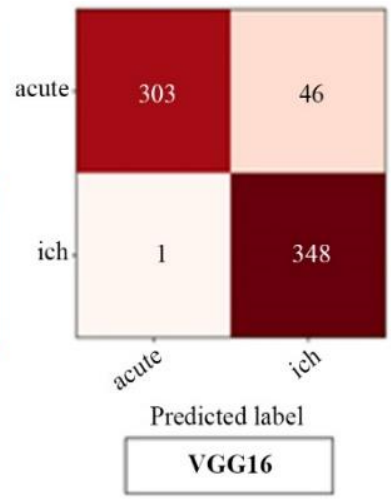

(c)

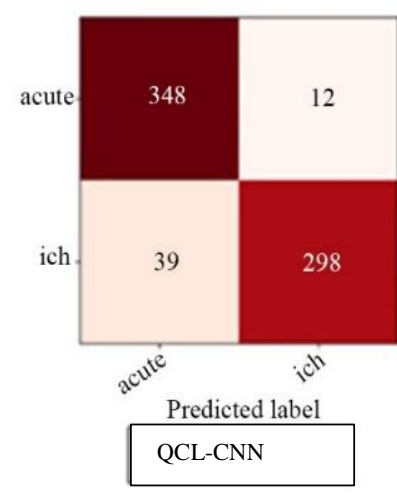

(d)

Figure. 3 Confusion matrix $1^{\text {st }}$ dataset of: (a) AlexNet, (b) ResNet50, (c) VGG16, (d) QCL-CNN, with 80 percent training and 20 percent testing.

Table 2. Comparison of the first data classification accuracy using 80 percent training and 20 percent testing

\begin{tabular}{lccccc}
\hline Method & Precision & TPR & FPR & F-measure & ACC (\%) \\
\hline AlexNet & 0.94 & 0.65 & 0.04 & 0.77 & 80.23 \\
ResNet50 & 0.90 & 0.71 & 0.08 & 0.79 & 81.52 \\
VGG16 & 0.94 & 0.90 & 0.01 & 0.94 & 94.27 \\
QCL-CNN & $\mathbf{0 . 9 6}$ & $\mathbf{0 . 9 6}$ & $\mathbf{0 . 0 4}$ & $\mathbf{0 . 9 6}$ & $\mathbf{9 5 . 9 1}$ \\
\hline \hline
\end{tabular}

K-fold cross-validation divides the sample randomly into $\mathrm{k}$ equal-sized sets. Each of the $\mathrm{k}$ shares contains a single set of validation data for testing the model, while the remaining $\mathrm{k}-1$ shares contain training data [29]. The procedure of crossvalidation is then performed $\mathrm{k}$ times, with each of the $\mathrm{k}$ sets being validated exactly once. The mean performance is then utilized to evaluate the method under consideration. This strategy is computationally intensive, but it fully exploits the entire collection of data, which is especially important when the sample size is very small. Additionally, this approach demonstrates how the trained model is generalizable to previously unknown data, avoiding the purposeful selection of data with superior test results [30].

\section{Results and discussion}

This study used two datasets for the experiment consisting of two and three classes that has been classified. The first dataset has ischemic and hemorrhage DW-MRI images. Meanwhile, in the second, another class was included together with these two image types, which contain DW-MRI of the normal brain. The image dataset classification using AlexNet and ResNet50 was only possible using a size of $224 \times 224 \times 3$.

\subsection{Results of the $1^{\text {st }}$ dataset}

The $1^{\text {st }}$ dataset processed with two experiments. The first experiment was carried out in this section to see if the proposed classification approach was effective. The image classification studies were determined by dividing DW-MRI images into 20 percent testing and 80 percent training sets for ischemic (acute) and hemorrhage (ich) conditions. Each category has 1742 images, which were further separated into 697 images for testing and 2787 images for training. The confusion matrix was created after several techniques of classification were used, as shown in Fig. 3, with ich and acute in the hemorrhage and ischemic classifications, respectively.

The numbers of correct and incorrect cases in the confusion matrix were shown in brown, and beige. The resulting confusion matrix of commonly used CNN architectures, namely AlexNet, ResNet50, and VGG16, are shown in Fig. 3 (a), 3 (b), and 3 (c). The various evaluation steps discussed in the previous section were also calculated for the classification method and are shown in Table 2. The mean classification accuracy obtained by AlexNet, ResNet50, and VGG16 was 80.3percent, 81.52percent, and 94.27 percent, respectively. However, the QCL-CNN accuracy classified is 95.91 percent. 


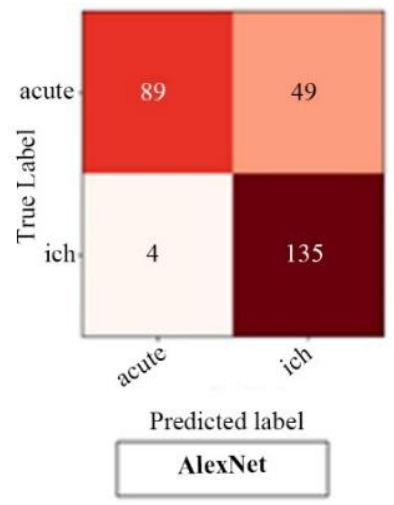

(a)

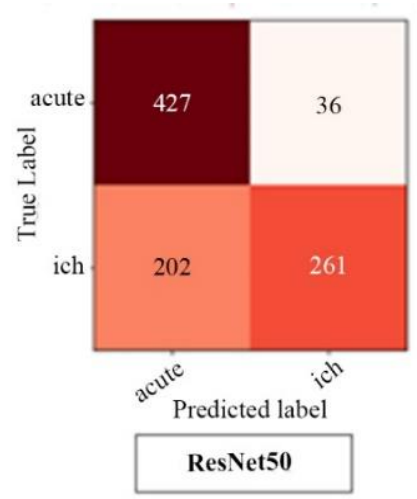

(b)

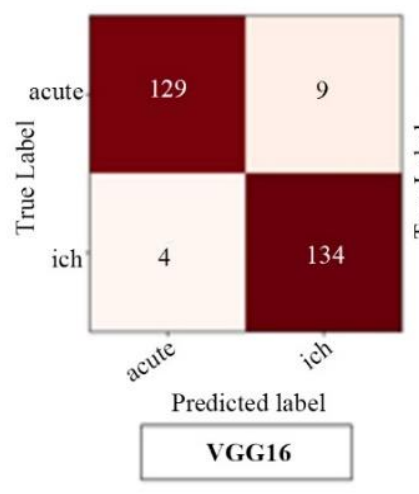

(c)

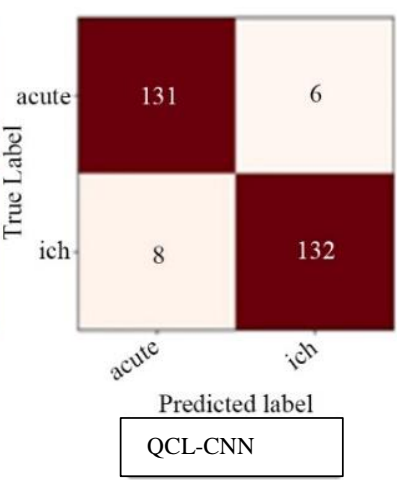

(d)

Figure. 4 Confusion matrix $1^{\text {st }}$ dataset of: (a) AlexNet, (b) ResNet50, (c) VGG16, (d) QCL-CNN, with 10-fold crossvalidation

Table 3. Comparison of Classify accuracy of $1^{\text {st }}$ dataset using 10 -fold cross-validation

\begin{tabular}{cccccc}
\hline Method & Precision & TPR & FPR & F-measure & ACC (\%) \\
\hline AlexNet & 0.91 & 0.78 & 0.07 & 0.84 & 85.20 \\
ResNet50 & 0.88 & 0.56 & 0.08 & 0.69 & 74.30 \\
VGG16 & 0.94 & 0.96 & 0.06 & 0.95 & 95.04 \\
QCL-CNN & $\mathbf{0 . 9 8}$ & $\mathbf{0 . 9 7}$ & $\mathbf{0 . 0 3}$ & $\mathbf{0 . 9 7}$ & $\mathbf{9 7 . 3 1}$ \\
\hline \hline
\end{tabular}

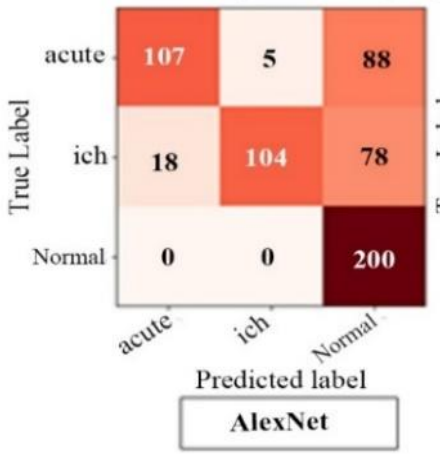

(a)

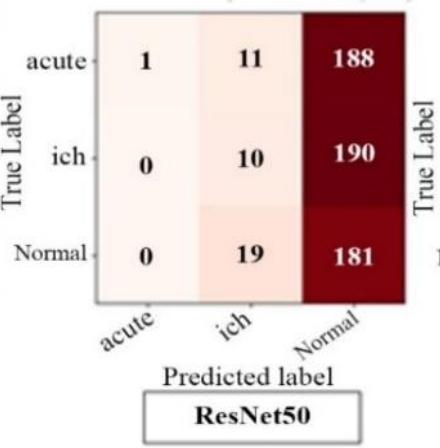

(b)

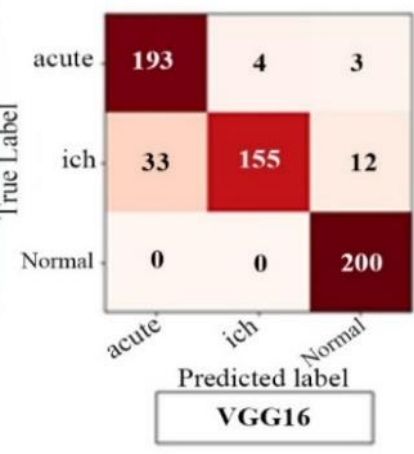

(c)

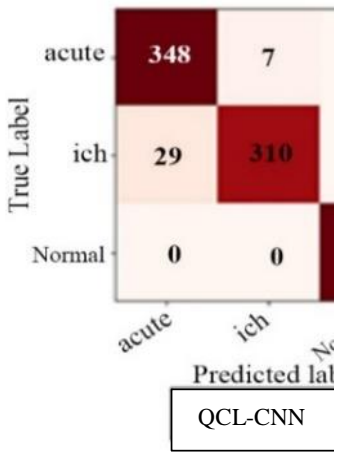

(d)

Figure. 5 Confusion matrix $2^{\text {nd }}$ dataset of: (a) AlexNet, (b) ResNet50, (c) VGG16, (d) QCL-CNN, with 80 percent training and 20 percent testing

In the second experiment with $1^{\text {st }}$ dataset , 10 -fold cross-validation was employed to determine the average categorization accuracy overall folds. The classification algorithms' confusion matrices are depicted in Fig. 4. Table 3 shows that QCL-CNN worked well in this trial, with an average accuracy of 98.77 percent. However, accuracies of 85.20 percent, 74.30 percent, 95 percent, and 97.31 percent were obtained using AlexNet, ResNet50, VGG18, and QCL-CNN. The confusion matrices make it easy to identify the classification outcomes for both experiments in terms of precision, TPR, FPR, and Fmeasure. Tables 2 and 3 illustrate the values derived by these performance measures. On a 20 percent testing dataset, the approach achieved average accuracy, TPR, FPR, and F-measure of 0.96, 0.96, 0.04 , and 0.96 , respectively. Their values in 10 fold cross-validation are $0.98,0.97,0.03$, and 0.97 , respectively.

\subsection{Result of the $2^{\text {nd }}$ Dataset}

The ischemic (acute), hemorrhage (ich), and normal DW MR images of the brain were used in this part, with each category containing 1742 images. Two experiments were also carried out by the authors. 
$\underline{\text { Table 4. Comparison of Classify accuracy of } 2^{\text {nd }} \text { dataset with } 80 \text { percent training and } 20 \text { percent testing }}$

\begin{tabular}{cccccc}
\hline Method & Precision & TPR & FPR & F-measure & ACC $(\%)$ \\
\hline AlexNet & 0.95 & 0.85 & 0.04 & 0.90 & 90.17 \\
ResNet50 & 0.88 & 0.53 & 0.07 & 0.66 & 72.93 \\
VGG16 & 0.95 & 0.92 & 0.05 & 0.93 & 93.25 \\
\hline QCL-CNN & $\mathbf{0 . 9 7}$ & $\mathbf{0 . 9 1}$ & $\mathbf{0 . 0 3}$ & $\mathbf{0 . 9 4}$ & $\mathbf{9 3 . 9 0}$ \\
\hline
\end{tabular}

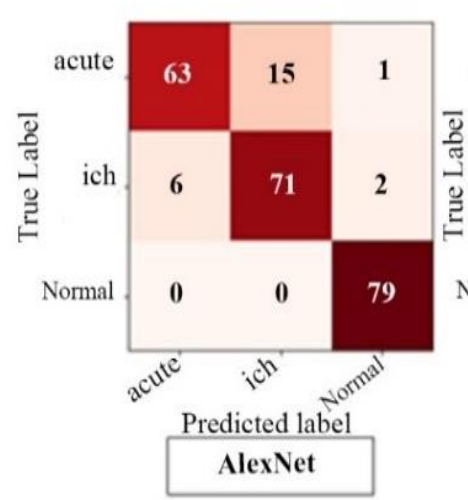

(a)

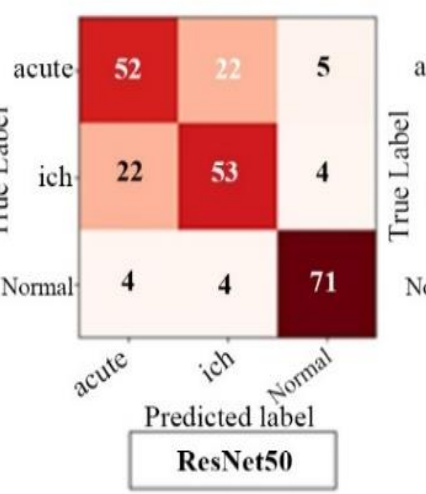

(b)

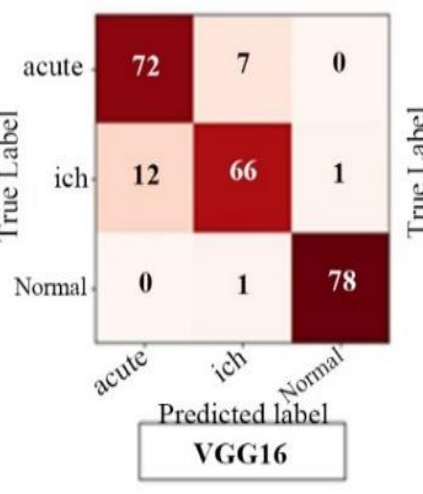

(c)

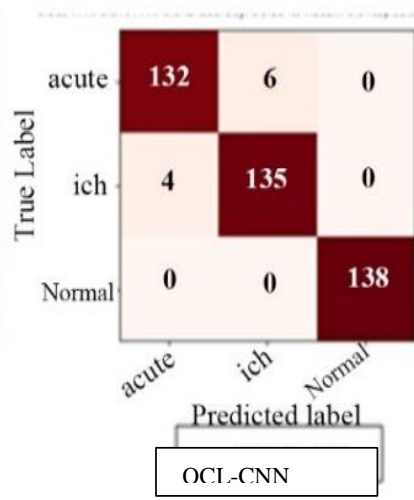

(d)

Figure. 6 Confusion matrix $2^{\text {nd }}$ dataset of: (a) AlexNet, (b) ResNet50, (c) VGG16, (d) QCL-CNN, with 10-fold crossvalidation.

The first was based on dividing the image dataset into an 80:20 training and testing ratio, while the others used ten-fold cross-validation on image datasets.

The first experiment for $2^{\text {nd }}$ dataset used 80 percent and 20 percent of the images obtained by dividing for training and testing, respectively. In Fig. 5 , confusion matrices show the correctly and incorrectly classified images of the dataset using various classification methods. A normal DW MR image of the brain is represented by $\mathrm{N}$ in the matrix. AlexNet, ResNet50, VGG16, and QCL-CNN achieved 90.17 percent, 72.93 percent, 93.25 percent, and 93.90 percent classification accuracy on the testing dataset, respectively, as shown in Table 4. Table 4 also includes other performance indicators such as precision, TPR, FPR, and F-measure. The proposed $\mathrm{CNN}$ delivers the best classification accuracy on three category datasets, with an accuracy of 93.90 percent, according to the experiment. Furthermore, just 36 DW MR images were incorrectly detected, compared to 190, 408, and 52 for AlexNet, ResNet50, and VGG16, respectively.

The second test using ten fold cross-validation technique of the 2 nd dataset of DW MRI. Fig. 6 shows the confusion matrices created after categorizing the three-category picture dataset. AlexNet, ResNet50, VGG16, and QCL-CNN have classification accuracy of 86.45 percent, 71.43 percent, 87.90 percent, and 94.96 percent, respectively. However, as seen in Table 5, QCL-CNN provides substantially superior accuracy.

\section{Discussion}

The numbers of successfully categorized cases utilizing the first experiment are shown in Fig. 3. The AlexNet classification revealed that 190 cases of hemorrhage (ich) stroke were accurately identified with a 54.44 percent accuracy. On the other hand, after correctly classifying 340 instances out of 349, the classification accuracy of ischemic (acute) stroke is 97.42 percent. Both categories had average accuracy, TPR, FPR, and F-measure of 0.94, 0.65, 0.04 , and 0.77 , respectively. The accuracy of image categorization with ResNet50 is 71.06 percent and 91.98 percent, respectively. The accuracy of image categorization using VGG16 for ich patients is 99.71 percent, whereas the accuracy for acute stroke cases is 86.82 percent.

The other evaluation metrics are $0.96,0.90,0.01$, and 0.0.94 for average precision, TPR, FPR, and Fmeasure for this dataset, respectively. For ich instances, image classification accuracy is 88.43 percent, whereas, for acute stroke cases, accuracy is 96.66 percent. For dataset 1, the QCL-CNN precision, TPR, FPR, and F-measure evaluations are 0.96, 0.96, 0.04 , and 0.96 , respectively. The total accuracy of QCL-CNN classification was 1.65percent higher 
than VGG16, 14.39 percent higher than ResNet50, and 15.18 percent higher than AlexNet.

In the second experiment, ten-fold crossvalidation was used to classify images from dataset one, as shown in Fig. 4. Furthermore, the classification accuracies for ICH and acute stroke images generated by AlexNet, ResNet50, and VGG16 are 97.12 percent and 65.49 percent, respectively. The ich and acute stroke accuracies of ResNet50 are 56, 37, and 92.22 percent, respectively. The ich and acute stroke accuracies in Model VGG16 are 97.10 percent and 92.75 percent, respectively. The accuracy of ich and acute strokes, when classified using QCL-CNN, is 96.35 percent and 95.6 percent, respectively. When classification is done with QCL-CNN, however, the system's overall accuracy is better than the other three approaches, with improvements of 12.11 percent, 23.01 percent, and 2.27 percent over AlexNet ResNet50 and VGG16, respectively. Table 3 shows the results of all other performance indicators, with the proposed approaches (QCL-CNN) having lower FPRs than the other three ways.

The second dataset describes the form of image classification with three categories of lower classification accuracy. The fundamental reason for this is that some of the situations are natural, such as ich, because white regions are comparable and contribute to the ich stroke's properties. Acute cases, on the other hand, are identified by the similarity of dark gray level pixels within the image. In confusion matrices, the number of cases incorrectly recognized as the other kind is represented by a deep brown color.

In the first experiment, assuming the number of accurately categorized images of each stroke is taken into account, a total of 104 (54percent) will be achieved. The chart also shows that 18 ich stroke images are classified as acute and 78 as normal, accounting for 9 percent and 39 percent of the 200

Table 5. Comparison of Classify accuracy of $1^{\text {st }}$ dataset with after 10-fold cross- validation of image

\begin{tabular}{cccccc}
\hline Method & Precision & TPR & FPR & F-measure & ACC(\%) \\
\hline AlexNet & 0.83 & 0.92 & 0.19 & 0.87 & 86.45 \\
ResNet50 & 0.67 & 0.83 & 0.40 & 0.74 & 71.43 \\
VGG16 & 0.90 & 0.85 & 0.09 & 0.87 & 87.90 \\
\hline QCL-CNN & $\mathbf{0 . 9 6}$ & $\mathbf{0 . 9 4}$ & $\mathbf{0 . 0 4}$ & $\mathbf{0 . 9 5}$ & $\mathbf{9 4 . 9 6}$ \\
\hline
\end{tabular}
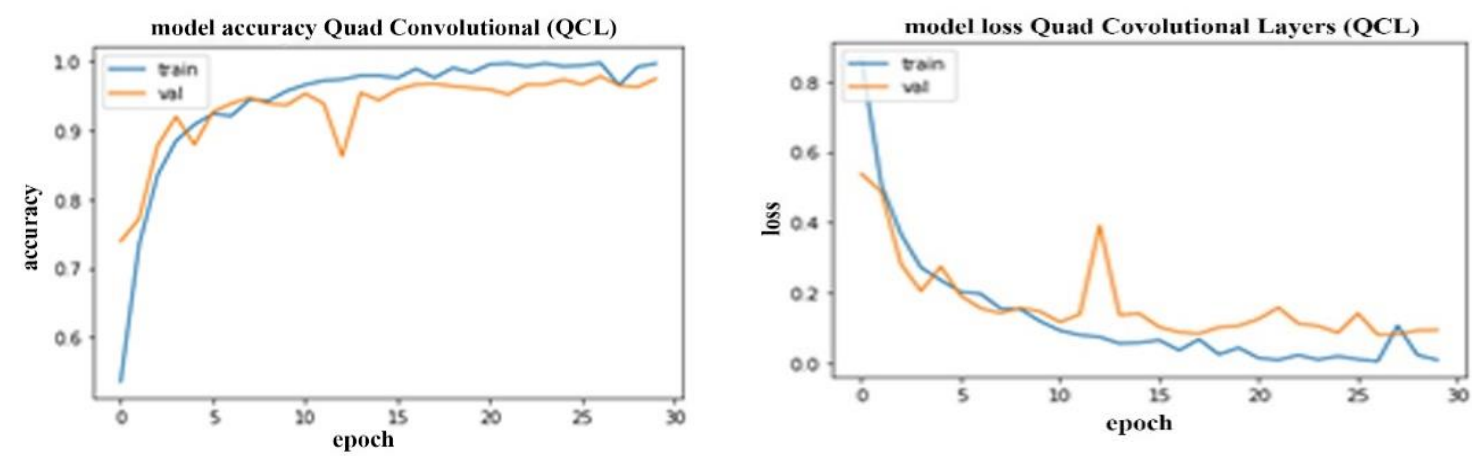

Figure. 7 Performance of the classification model on $224 \times 224 \times 3$ data size ( 1 st dataset )
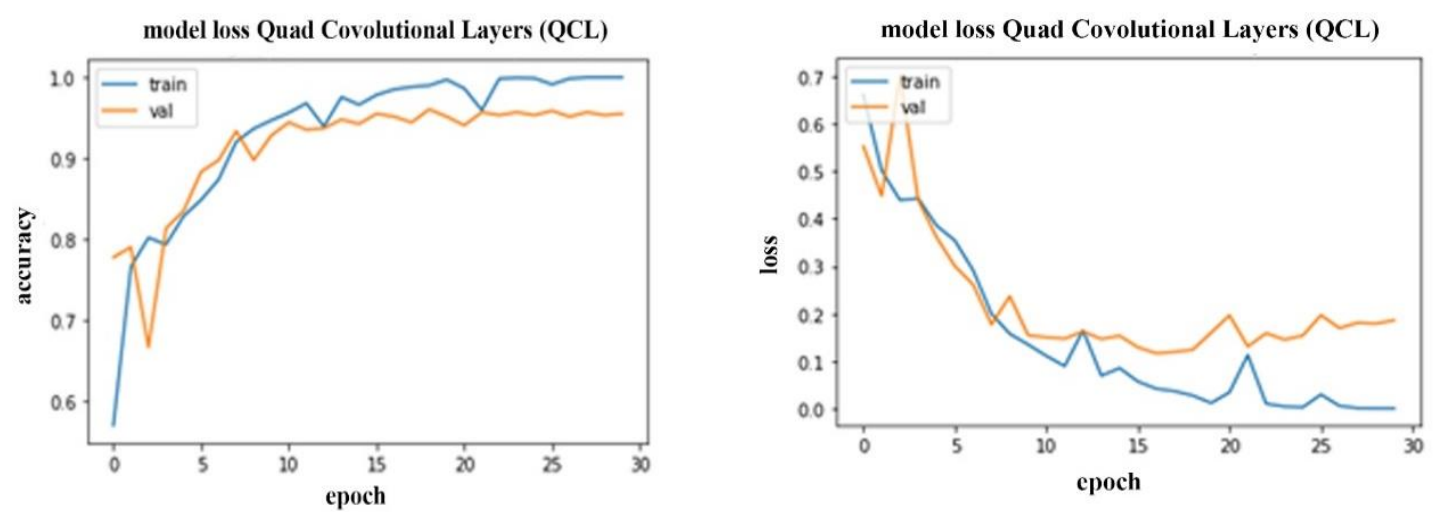

Figure. 8 Performance of the classification model on $224 \times 224 \times 3$ data size ( 2 nd dataset ) 
Table 6. Experiment results with DW-MRI researcher data (2nd dataset, $\mathrm{n}=5226$ )

\begin{tabular}{llcc}
\hline \multicolumn{1}{c}{ Prior Work } & \multicolumn{1}{c}{ Network } & $\begin{array}{c}\text { Number of } \\
\text { Convolution } \\
\text { Layers }\end{array}$ & ACC (\%) \\
\hline Do et al. [31] & $\begin{array}{l}\text { Recurrent Residual Convolutional } \\
\text { Neural Network (RRCNN) } \\
\text { Cross-Modal Convolutional Neural } \\
\text { Zhu, H et al. [32] }\end{array}$ & 12 & 92.31 \\
H. Kim et al [33] & 3D Convolutional Neural Network & 7 & 91.78 \\
$\begin{array}{l}\text { Quad Convolutional } \\
\text { Layers (QCL)-CNN }\end{array}$ & Quad Convolutional Layers & 13 & 90.30 \\
\hline \hline
\end{tabular}

images, respectively. The percentage of acute and normal DW MR pictures properly classified is $\mathbf{5 3 . 5 0}$ percent and 100 percent, respectively. Similarly, when employing the ResNet50 approach, the classification accuracy for ich and acute strokes is 5 percent and 0.5 percent, respectively, whereas the classification accuracy for normal pictures is 90.5 percent, as shown in Fig. 4. (b). In Fig. 4 (c), the VGG16 method shows that the ich and acute strokes accuracies are 77.5percent, and 96.5percent with normal images classification of 100percent. The images classified by the authors using the proposed layers (QCL-CNN) have ich and acute strokes of 91.44percent and 95.34percent, with 100percent correctly classified normal images as shown Fig. 4 (d). AlexNet, ResNet50, and VGG16 all performed better than AlexNet, ResNet50, and VGG16 in classifying ich, acute, and normal images. As demonstrated in Table 4, the average accuracy of QCL-CNN is higher than that of others.

Experiments performed on three categories of image datasets using the 10 fold cross-validation in $2^{\text {nd }}$ dataset, shows that the ich stroke is 89.87 percent by identifying 71 images correctly out of 79 . The number of acute images correctly identified is 63 (79.75percent) of total acute images, and 100percent of normal images. When performed with ResNet50, the classification accuracies of ich and acute strokes are 67.08percent, and 65.83percent, while the normal images are 89.87percent. Likewise, when using the VGG16 method, ich and acute strokes' classification are 83.54percent and 91.14 percent, while 98.73percent was obtained for the normal images, as shown in Fig. 6 (c). When classified by the QCL$\mathrm{CNN}$, the classification accuracies obtained for ich and acute strokes are 97.12percent, and 95.65percent, while 100percent was used for normal images. Based on these accuracies, the overall classification of the QCL-CNN has also been improved by 8.51percent, 23.53percent, and 7.06percent over AlexNet, ResNet50, and VGG16 respectively, as shown in Table 5.
Methods including data augmentation, learning rate variation, and annealing were used to help fit the large dataset into the deep convolutional neural network architecture, as discussed above. This was done to produce significant results, as shown in Fig. 8. Training loss $=0.0098$, training accuracy $=0.9970$, validation loss: 0.0946, and validation accuracy: 0.9749 are the final results $\left(1^{\text {st }}\right.$ dataset $)$. For $2^{\text {nd }}$ dataset, the training accuracy was 1.0000 , the training loss was 5.4236e-04, the validation loss was 0.1861 , and the validation accuracy was 0.9552 .

The proposed method is also compared to Do et al.[31] research, which similarly classified stroke images. In stroke care, the proposed early diagnosis and rapid quantification of acute ischemic lesions are critical. DWI datasets are showing acute anterior circulation stroke. The classification method used in this study is Repeated Residual Convolution Neural Network (RRCNN). A pre-trained VGG16 and Inception V3 employs twelve convolution layers. $\mathrm{Zhu}, \mathrm{H}$ et al.[32] suggested an automatic machine learning technique. They first create a cross-modal convolutional neural network that can accurately detect stroke lesions from DWI and FLAIR images, employing seven convolution layers. Kim et al. [33] The 3D Convolutional neural network method was used in this study using Fluid-Attenuated Inversion Recovery (FLAIR) MRI data with employs thirteen convolution layers.

The dataset used in the study, with a total of 5226 images (2nd dataset). Then, the data was tested on the convolution layer model from the previous researchers (Table 6).

The results of these experiments are written in Table 6. Do et al. [31] adopted the VGG16 and Resnet, employing 12 convolution layers, and the accuracy result shows 92.31 percent. As for $\mathrm{Zhu}, \mathrm{H}$ et al. [32], which employs seven convolution layers, the accuracy result is 91.78 percent. The research conducted by $\mathrm{H}$. Kim et al. [33] adopted 13 convolution layers, and the accuracy result has a value of 90.30 percent. In this study, we used only 
four convolution layers in the proposed Quad Convolutional Layers (QCL)-CNN, and the experiment deliver the highest accuracy value 93.90 percent.

\section{Conclusion}

This study introduces a new classification on brain stroke injury at DW MR images, by employing Quad Convolution Layer adapted in CNN.

The new QCL-CNN architecture model can be used for recognising the dissimilar between the first brain stroke injury type ( ICH and acute)/ $1^{\text {st }}$ data set and second brain stroke injury type ( $\mathrm{ICH}$, acute and normal)/ $2^{\text {nd }}$ data set .

The performance of QCL-CNN model architecture assessed into two testing. The first experiment used an image dataset split into 20 percent testing and 80 percent training. Then, ten-fold cross-validations were performed in the second experiment.

This study also comparing the QCL-CNN with others $\mathrm{CNN}$ architectures such as AlexNet, ResNet50, and VGG16. The results showed that QCL-CNN is performing excellent from those method on data set DW MR image.

\section{Acknowledgments}

The author would like to thank the Ministry of Research, Technology and Higher Education of the Republic of Indonesia for supporting this research through Indonesian Education Scholarship (BPPDN). Furthermore, the authors also gratefull to the Gatot Subroto Army Hospital, the Department of Radiology, the Universitas Airlangga Hospital for providing MRI data for stroke patients, and the University Center of Excellence on Artificial Intelligence for Healthcare and Society (UCE AIHeS). In addition, this study was partially funded by the Education Fund Management Institute (LPDP) under the Innovative Productive Research Grant (RISPRO) scheme - Invitation 2019, contract number: PRJ-41/LPDP/2019.

\section{Conflicts of interest}

The authors declare no conflict of interest.

\section{Author contributions}

Conceptualization: Andi Kurniawan Nugroho; Methodology: Andi Kurniawan Nugroho; Software: Andi Kurniawan Nugroho; Validation: Terawan Agus Putranto; Formal analysis: Andi Kurniawan
Nugroho; Writing original draft preparation: Andi Kurniawan Nugroho; Writing review and editing: Terawan Agus Putranto, I Ketut Eddy Purnama and Mauridhi Hery Purnomo;Visualization: Mauridhi Hery Purnomo; Supervision: Mauridhi Hery Purnomo;Funding acquisition: I Ketut Eddy Purnama.

\section{References}

[1] J. T. Marbun, Seniman, and U. Andayani, "Classification of stroke disease using convolutional neural network", J. Phys. Conf. Ser., Vol. 978, No. 1, 2018, doi: 10.1088/17426596/978/1/012092.

[2] U. R. Acharya, K. M. Meiburger, O. Faust, J. E. W. Koh, S. L. Oh, E. J. Ciaccio, A. Subudhi, V. Jahmunah, and S. Sabut, "Automatic detection of ischemic stroke using higher order spectra features in brain MRI images", Cognitive Systems Research, Vol. 58, pp. 134-142, 2019, doi: 10.1016/j.cogsys.2019.05.005.

[3] K. J. V. Everdingen, J. V. D. Grond, L. J. Kappelle, L. M. P. Ramos, and W. P. T. M. Mali, "Diffusion-weighted magnetic resonance imaging in acute stroke", Stroke, Vol. 29, No. 9, pp. 1783-1790, 1998, doi: 10.1161/01.STR.29.9.1783.

[4] W. Reith, Y. Hasegawa, L. L. Latour, B. J. Dardzinski, C. H. Sotak, and M. Fisher, "Multislice diffusion mapping for 3D evolution of cerebral ischemia in a rat stroke model", Neurology, Vol. 45, No. 1, pp. 172-177, 1995, doi: 10.1212/WNL.45.1.172.

[5] T. Back, M. H. Berlage, K. Kohno, and K. A. Hossmann, "Diffusion nuclear magnetic resonance imaging in experimental stroke correlation with cerebral metabolites", Stroke, Vol. 25, No. 2, pp. 494-500, 1994, doi: 10.1161/01.STR.25.2.494.

[6] A. G. Sorensen, F. S. Buonanno, R. G. Gonzalez, L. H. Schwamm, M. H. Lev, F. R. H. Helbinger, T. G. Reese, R. M. Weisskoff, T. L. Davis, MS, N. Suwanwela, U. Can, J. A. Moreira, W. A. Copen, R. B. Look, B. A. S. P. Finklestein, B. R. Rosen, W. J. Koroshetz, "Hyperacute stroke: evaluation with combined multisection diffusion-weighted and hemodynamically weighted echo-planar MR imaging", Radiology, Vol. 199, No. 2, pp. 391-401, 1996, doi: 10.1148/radiology.199.2.8668784.

[7] M. Matešin, L. Sven, and D. Petravić, "A RuleBased Approach to Stroke Lesion Analysis from CT Brain Images", Int. Symp. Image Signal Process. Anal. ISPA, Vol. 2001-Janua, pp. 219223, 2001, doi: 10.1109/ISPA.2001.938631. 
[8] S. A. Peixoto and P. P. R. Filho, "NeurologistLevel Classification of Stroke Using a Structural Co-Occurrence Matrix Based on The Frequency Domain", Comput. Electr. Eng., Vol. 71, No. April, pp. 398-407, 2018, doi: 10.1016/j.compeleceng.2018.07.051.

[9] W. L. Nowinski, G. Qian, and D. F. Hanley, "A CAD System for Hemorrhagic Stroke", Neuroradiol. J., Vol. 27, No. 4, pp. 409-416, 2014, doi: 10.15274/NRJ-2014-10080.

[10] Z. Mousavi, T. Y. Rezaii, S. Sheykhivand, A. Farzamnia, and S. N. Razavi, "Deep Convolutional Neural Network for Classification of Sleep Stages from SingleChannel EEG Signals", J. Neurosci. Methods, Vol. 324, No. December 2018, 2019, doi: 10.1016/j.jneumeth.2019.108312.

[11] E. Başaran, Z. Cömert, and Y. Çelik, "Convolutional neural network approach for automatic tympanic membrane detection and classification", Biomed. Signal Process. Control, Vol. 56, 2020, doi: 10.1016/j.bspc.2019.101734.

[12] H. I. Suk, S. W. Lee, and D. Shen, "Hierarchical Feature Representation and Multimodal Fusion with Deep Learning for AD/MCI Diagnosis", Neuroimage, Vol. 101, pp. 569-582, 2014, doi: 10.1016/j.neuroimage.2014.06.077.

[13] S. Hochreiter, "Long Short-Term Memory", Neural Comput., Vol. 1780, pp. 1735-1780, 1997.

[14] F. Ouhmich, V. Agnus, V. Noblet, F. Heitz, and P. Pessaux, "Liver Tissue Segmentation in Multiphase CT Scans Using Cascaded Convolutional Neural Networks", Int. J. Comput. Assist. Radiol. Surg., Vol. 14, No. 8, pp. 12751284, 2019, doi: 10.1007/s11548-019-01989-z.

[15] G. E. Hinton, S. Osindero, and Y. W. Teh, "A Fast Learning Algorithm for Deep Belief Nets", Neural Comput., Vol. 18, pp. 1527-1554, 2006.

[16] C. H. Wan, L. H. Lee, R. Rajkumar, and D. Isa, "A Hybrid Text Classification Approach with Low Dependency on Parameter by Integrating K-Nearest Neighbor and Support Vector Machine", Expert Syst. Appl., Vol. 39, No. 15, pp. 11880-11888, 2012, doi: 10.1016/j.eswa.2012.02.068.

[17] A. Subudhi, M. Dash, and S. Sabut, "Automated Segmentation and Classification of Brain Stroke Using Expectation-Maximization and Random Forest Classifier", Biocybern. Biomed. Eng., Vol. 40, No. 1, pp. 277-289, 2020, doi: 10.1016/j.bbe.2019.04.004.

[18] M. Toğaçar, Z. Cömert, and B. Ergen, "Classification of Brain MRI Using Hyper Column Technique with Convolutional Neural
Network and Feature Selection Method", Expert Syst. Appl., Vol. 149, p. 113274, 2020, doi: 10.1016/j.eswa.2020.113274.

[19] I. Guyon, "Gene Selection for Cancer Classification”, pp. 389-422, 2002.

[20] S. R. Amendolia, G. Cossu, M. L. Ganadu, B. Golosio, G. L. Masala, and G. M. Mura, "A comparative Study of K-Nearest Neighbour, Support Vector Machine and Multi-Layer Perceptron for Thalassemia screening", Chemom. Intell. Lab. Syst., Vol. 69, pp. 13-20, 2003, doi: 10.1016/S0169-7439(03)00094-7.

[21] A. Bansal, C. Castillo, R. Ranjan, and R. Chellappa, "The do's and don'ts for CNN-Based Face Verification", In: Proc. of 2017 IEEE International Conference on Computer Vision Workshops, pp. 2545-2554, 2017, doi: 10.1109/ICCVW.2017.299K.

[22] K. E. Saatman, A. C. Duhaime, R. Bullock, A. I. R. Maas, A. Valadka, and G. T. Manley, "Classification of Traumatic Brain Injury for Targeted Therapies", Journal of Neurotrauma, Vol. 25, No. 7, pp. 719-738, 2008, doi: 10.1089/neu.2008.0586.

[23] F. T. Mozqueda, J. He, I. B. Yeh, L. H. Schwamm, M. H. Lev, P. W. Schaefer, and R. G. González, "An Acute Ischemic Stroke Classification Instrument That Includes CT or MR Angiography: The Boston Acute Stroke Imaging Scale", AJNR Am J Neuroradiol, Vol. 29, No. 6, pp. 1111-1117, 2008, doi: 10.3174/ajnr.A1000.

[24] S. Anbumozhi, "Computer-aided detection and diagnosis methodology for brain stroke using adaptive neuro-fuzzy inference system classifier", Int. J. Imaging Syst. Technol., Vol. 30, No. 1, pp. 196-202, 2020, doi: 10.1002/ima.22380.

[25] A. Gautam, B. Raman, and S. Raghuvanshi, "A hybrid approach for the delineation of brain lesion from CT images", Biocybern. Biomed. Eng., Vol. 38, No. 3, pp. 504-518, 2018, doi: 10.1016/j.bbe.2018.04.003.

[26] A. Gautam and B. Raman, "Local Gradient of Gradient Pattern: a Robust Image Descriptor for The Classification of Brain Strokes from Computed Tomography Images", Pattern Anal. Appl., Vol. 23, No. 2, pp. 797-817, 2020, doi: 10.1007/s10044-019-00838-8.

[27] A. Krizhevsky, I. Sutskever, and G. E. Hinton, "ImageNet Classification with Deep Convolutional Neural Networks", Commun. ACM, Vol. 60, No. 6, pp. 84-90, 2017, doi: $10.1145 / 3065386$.

[28] G. E. Hinton, "Rectified Linear Units Improve 
Restricted Boltzmann Machines", No. 3.

[29] I. B. Santoso, Y. Adrianto, A. D. Sensusiati, D. P. Wulandari, and I. K. E. Purnama, "Epileptic EEG Signal Classification Using Convolutional Neural Network Based on Multi-Segment of EEG Signal", Int. J. Intell. Eng. Syst., Vol. 14, No. 3, pp. 160-176, 2021, doi: 10.22266/ijies2021.0630.15.

[30] K. Men, H. Geng, C. Cheng, H. Zhong, M. Huang, Y. Fan, J. P. Plastaras, A. Lin, and Y. Xiao, "Technical Note: More accurate and efficient segmentation of organs - at - risk in radiotherapy with convolutional neural networks cascades", Med. Phys., p. mp.13296, 2018, doi: 10.1002/mp.13296.

[31] L. N. Do, B. H. Baek, S. K. Kim, H. J. Yang, I. Park, and W. Yoon, "Automatic Assessment of ASPECTS Using Diffusion-Weighted Imaging in Acute Ischemic Stroke Using Recurrent Residual Convolutional Neural Network", Diagnostics, Vol. 10, No. 10, 2020, doi: 10.3390/diagnostics10100803.

[32] H. Zhu, L. Jiang, H. Zhang, L. Luo, Y. Chen, and Y. Chen, "An Automatic Machine Learning Approach for Ischemic Stroke Onset Time Identification Based on DWI and FLAIR Imaging", NeuroImage Clin., Vol. 31, p. 102744, 2021, doi: 10.1016/j.nicl.2021.102744.

[33] H. Kim, Y. Lee, Y. H. Kim, Y. M. Lim, J. S. Lee, J. Woo, S. K. Jang, Y. J. Oh, H. W. Kim, E. J. Lee, D. W. Kang, and K. K. Kim, "Deep Learning-Based Method to Differentiate Neuromyelitis Optica Spectrum Disorder From Multiple Sclerosis", Front. Neurol., Vol. 11, p. 599042, 2020, doi: 10.3389/fneur.2020.599042. 